\title{
Self-oscillation in electrochemical transistors: An RLC modeling approach
}

\author{
Deyu Tu and Robert Forchheimer
}

\section{Linköping University Post Print}

N.B.: When citing this work, cite the original article.

Original Publication:

Deyu Tu and Robert Forchheimer, Self-oscillation in electrochemical transistors: An RLC modeling approach, 2012, Solid-State Electronics, (69), 7-10.

http://dx.doi.org/10.1016/j.sse.2011.12.006

Copyright: Elsevier

http://www.elsevier.com/

Postprint available at: Linköping University Electronic Press

http://urn.kb.se/resolve?urn=urn:nbn:se:liu:diva-76532 


\title{
Self-oscillation in electrochemical transistors: An RLC modeling
}

\author{
approach \\ Deyu Tu, Robert Forchheimer* \\ Information Coding, ISY, Linköping University, SE-581 83, Linköping, Sweden
}

\begin{abstract}
We propose an RLC model for PEDOT:PSS electrochemical transistors to interpret the persistent oscillating currents observed in experiments. The electrochemical reaction is represented by an inductor in the equivalent circuit. The simulation results show that an electrochemical device can be operated as normal transistors or oscillators under different voltage bias. This model predicts that analog circuit functions can be realized with "inductor-like" electrochemical devices.
\end{abstract}

\section{Highlights}

$>$ Self-starting current oscillation in polymer electrochemical devices is modeled by an RLC equivalent circuit.

$>$ The simulation results show good agreement with the experimental data.

This model indicates that electrochemical devices are promising for analog circuit functions and even artificial biological systems.

Key words: electrochemical transistors; negative capacitance; PEDOT:PSS; RLC circuit; selfoscillation.

\footnotetext{
* Corresponding author at: Information Coding, ISY, Linköping University, SE-581 83, Linköping, Sweden. Tel.: +46 013 281342; fax: +46 013281342 .

E-mail addresses: robert@isy.liu.se (R. Forchheimer), deyu@isy.liu.se (D. Tu).
} 


\section{Introduction}

Conjugated polymers have been highly attractive and heavily investigated for potential applications in electronics, due to their unique properties, such as flexibility, tunable functionality, and low-cost production [1, 2]. Among numerous organic/polymer electronic devices, transistors have been demonstrated, since they are the most basic components to realize circuit functions. Using a conducting polymer blend poly(3,4-ethylene-dioxythiophene):poly(styrene sulphonic acid) (PEDOT:PSS), electrochemical transistors have been reported, exhibiting a fairly low operating voltage, large channel current and printability [3, 4]. Unlike field effect transistors, the channel current in electrochemical transistors is modulated by gate voltage via a redox process, due to the variable conductivity of PEDOT:PSS at different redox states. The operation mechanism in the electrochemical transistors is a complex combination of electronic transport, ionic transport and electrochemical reaction. The complexity of this process makes it challenging to develop physical models to simulate the device behaviors precisely, especially for unexpected phenomenon, such as current oscillations. There are a few examples reporting current oscillations in polymeric electronic devices, including polyaniline (PANI) transistors [5] and bistable devices [6]. Both of them explained the oscillations by $\mathrm{RC}$ equivalent circuits with variable resistance and a number of assumptions.

In this work, a compact RLC circuit model is proposed to mimic the behaviors of PEDOT:PSS electrochemical transistors. An "inductor-like" component is introduced to this model based on the energy conversion in the electrochemical process. The persistent oscillating currents observed in experiments can be interpreted by this model as expected. The simulation results show good agreement with experimental data. Besides oscillators, the electrochemical device can be operated as normal transistors as well at certain voltage bias.

\section{Experimental}


The PEDOT:PSS electrochemical transistors were fabricated as reported [3], with a layout illustrated in the inset of Fig. 1. The PEDOT:PSS (blue area) was used as received from AGFAGeavert. The electrolyte (light yellow) was deposited by spin-coating with $0.5 \mathrm{M} \mathrm{Na}_{2} \mathrm{SO}_{4}$ aqueous solution. The channel area was $250 \mu \mathrm{m} \times 500 \mu \mathrm{m}$. The electrical measurements were performed in an ambient atmosphere with a Keithley 2400 Sourcemeter and HP power supply (E3261A) controlled using Labview software. The typical output characteristics with oscillating currents are presented in Fig. 1. The drain voltage $\left(V_{D}\right)$ swept from 0 to $2 \mathrm{~V}$ with a step of $0.005 \mathrm{~V}$ at a rate of 1 $\mathrm{V} / \mathrm{s}$, while the gate voltage $\left(V_{G}\right)$ was kept from 1 to $2.2 \mathrm{~V}$ with a step of $0.2 \mathrm{~V}$, respectively. From this figure, it is seen that the device showed apparent current oscillation at some certain voltage bias.

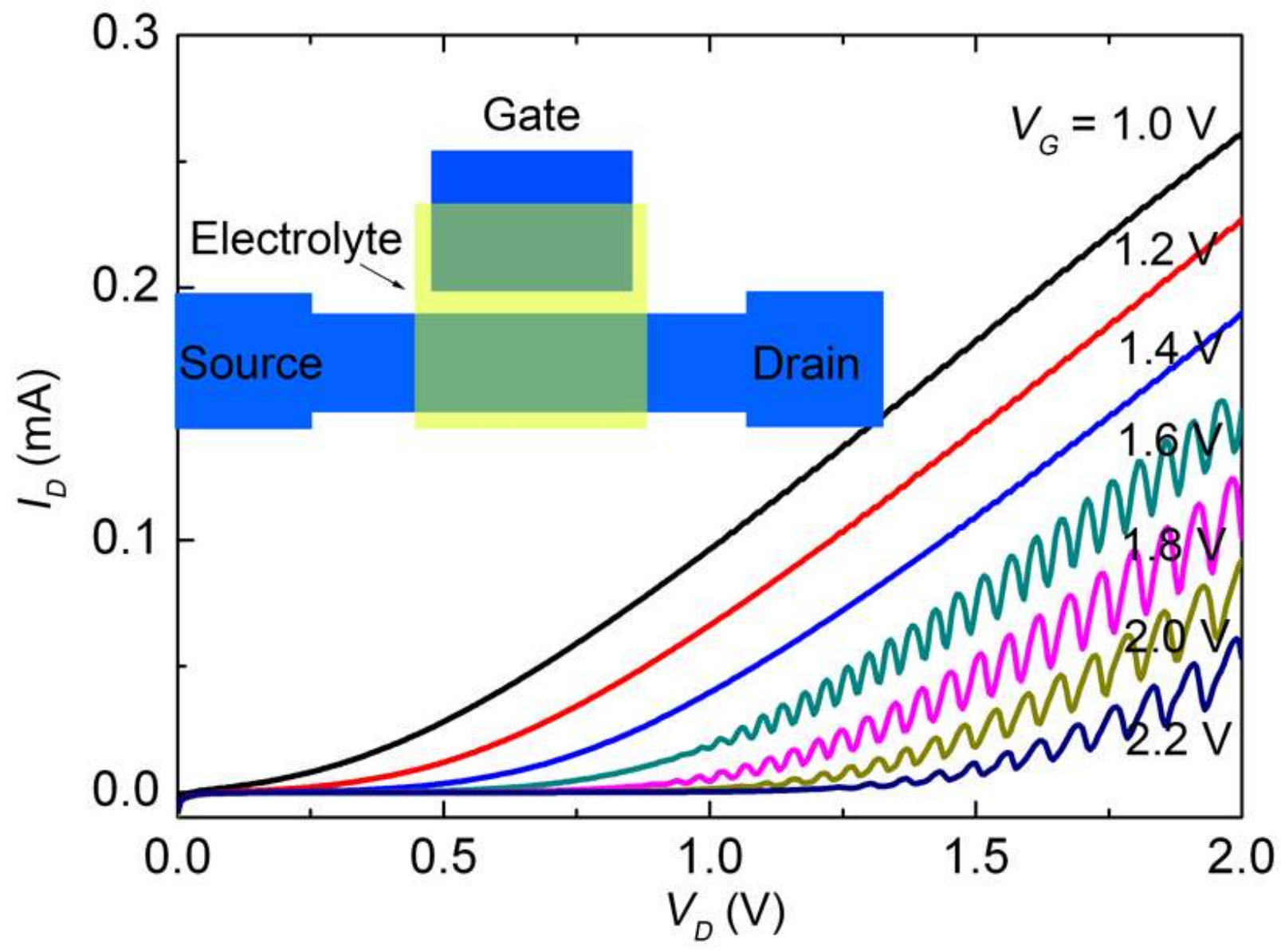

Fig. 1 The output characteristics of PEDOT:PSS electrochemical transistors, exhibiting self-starting current oscillation. Inset: the schematic of the transistor geometry. 


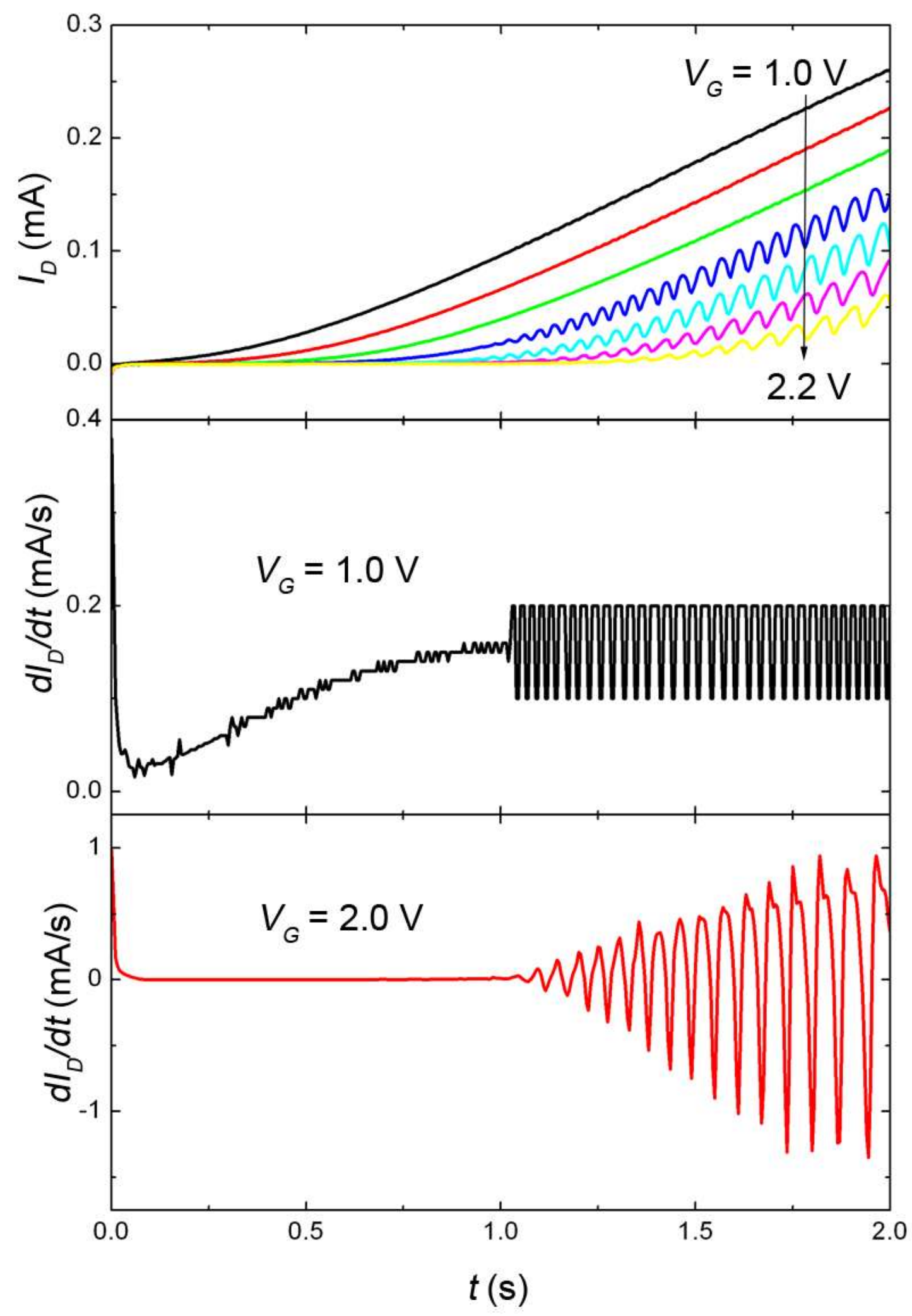

Fig. 2 (a) The time-resolved current at various gate voltage, converted from the data in Fig. 1. The current differential to time at $V_{G}=1 \mathrm{~V}$ (b) and $2 \mathrm{~V}$ (c), respectively. 
To study these data from another aspect, we convert the current versus voltage curves to time-resolved plots shown in Fig. 2(a), considering the drain voltage sweeping rate is $1 \mathrm{~V} / \mathrm{s}$. By differentiating the currents in Fig. 2(a) to time, the current variation upon time is obtained. Here, two curves at $V_{G}=1.0$ and $2.0 \mathrm{~V}$ are selected as examples and shown in Fig. 2(b)\&(c). From this graph, there are a few common features that can be seen, such as i) self-starting oscillation; ii) amplitude is dependent on gate voltage; and iii) the mean currents of oscillation are approximately linear to time/drain voltage. To simulate these behaviors, an RLC model is proposed and studied below.

\section{Model Theory and Derivation}

The conductivity of polymer PEDOT:PSS can be modulated through a redox process in the presence of ion transport via the following reaction:

$$
\mathrm{PEDOT}^{+} \mathrm{PSS}^{-}+\mathrm{M}^{+}+e^{-} \leftrightarrow \mathrm{PEDOT}^{0}+\mathrm{M}^{+} \mathrm{PSS}^{-}
$$

The cation $\mathrm{M}^{+}$in an electrolyte layer assists the reduction process, driven by the gate voltage, which reduce the conductivity of PEDOT:PSS, as shown in Fig. 3(b). While electrochemistry occurs in the device, the electrical energy is converted to chemical energy. This process is reversible. As known, a similar energy conversion between electric and magnetic field occurs when current passes through an inductor. Furthermore, "inductor-like" negative capacitance is often observed in organic light emitting diodes $[7,8]$ and it is believed that this is due to the dynamic effect of energy distribution on occupancy/release of interfacial states at the electrode/organic interface [9] or originating from accumulation and recombination of carriers near the organic/organic interface [10]. For electrochemical transistors, it is possible to have similar phenomenon to negative capacitance, although the underlying mechanism could be different. At the redox kinetics, there are two kinds of charge/ion transfer processes in electrochemical transistors. One is electron/ion transfer at the interface, and the other is charge transport within the channel. The 
faradiac current at the interface could form a current-driven negative impedance element in the device, and the energy conversion takes place during the redox process at the same time. To represent negative capacitance so-called inductive effect, equivalent circuits often contain inductance rather than real capacitance with negative value that leads to capacitance neutralization if there is positive capacitance [11]. This discussion implies that it is highly likely that there is an inductor-like element acting as an energy reservoir in electrochemical devices, besides capacitors. Hence, from the viewpoint of energy exchange, we use an inductor module as an analog in our electrochemical device model, although it is not exactly the same as conventional inductors realized by metal coil.

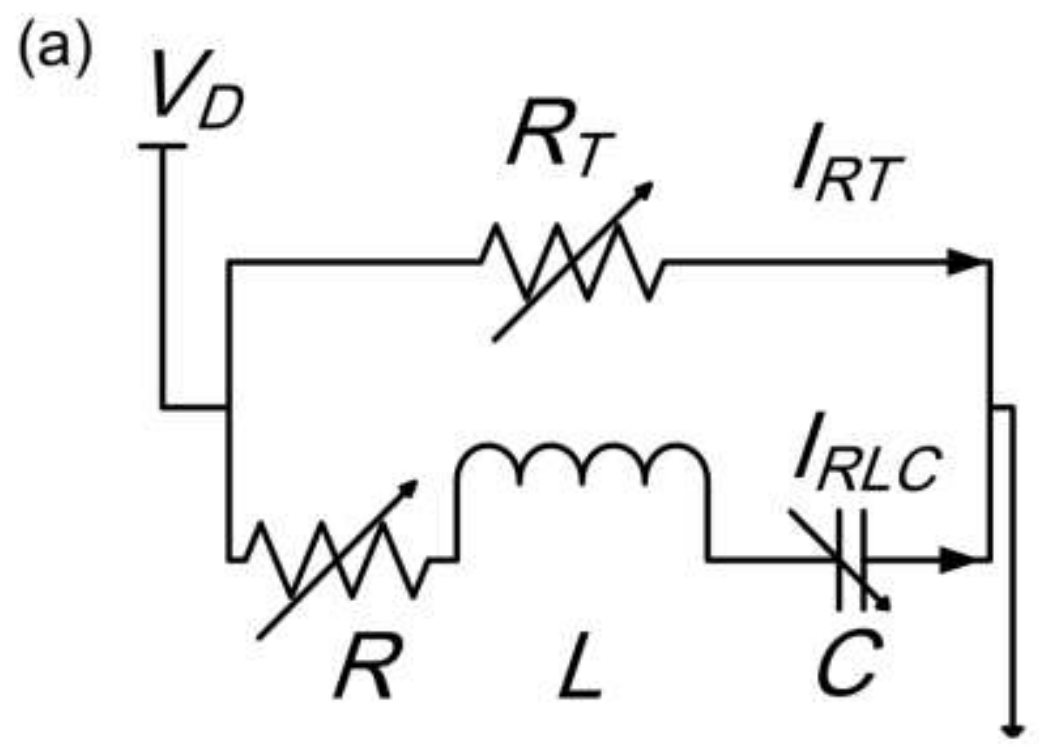

(b)

Electrolyte

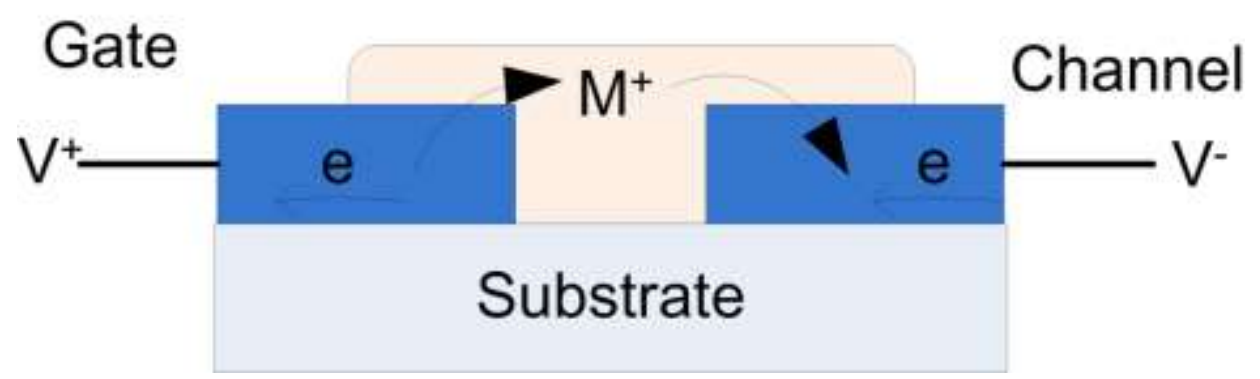

Fig. 3 (a) The equivalent circuit of proposed RLC model in this work. (b) The illustrated ion/electron transport in an electrochemical cell. 
The proposed equivalent circuit of an electrochemical device is presented in Fig. 3(a). The $R_{T}$ is the channel resistance dependent on gate voltage $V_{G}$, and the $R, L, C$ are the resistor, inductor and capacitor in the serial RLC module, respectively. The drain voltage $V_{D}$ is applied to the drain electrode, while the source is grounded. In our simulation, the drain current $\left(I_{D}\right)$ is calculated and plotted at certain conditions that $V_{G}$ is kept at fixed voltage while $V_{D}$ sweep at the same rate as that used in experimental measurements. We assume that each current curve is independent from the rest for multi- $V_{G}$ simulation, to simplify the simulation.

To obtain $I_{D}$ of the equivalent circuit, two branch currents ( $I_{R L C}$ and $I_{R T}$, referred below) have to be calculated respectively, and then summed together, where $I_{R L C}$ is the current through the RLC module and $I_{R T}$ is the channel current modulated by the gate voltage.

Applying Kirchhoff's voltage law to the equivalent circuit in Fig. 3(a), we have

$$
V_{R_{T}}=V_{R}+V_{L}+V_{C}=V_{D}
$$

where $V_{R}, V_{L}, V_{C}, V_{R T}$ are the voltages across the elements $R, L, C, R_{T}$, respectively.

Substituting the equation above with the three voltages $V_{R}, V_{C}, V_{L}$ in the RLC branch,

$$
L \frac{d I_{R L C}}{d t}+I_{R L C} R+\frac{1}{C} \int_{-\infty}^{\tau} I_{R L C}(\tau) d \tau=V_{D}
$$

Differentiating Eq. (3) and dividing by $L$, a second order differential equation on $I_{R L C}$ is written as

$$
\frac{d^{2} I_{R L C}}{d t^{2}}+2 \alpha \frac{d I_{R L C}}{d t}+\omega_{0}^{2} I_{R L C}-1=0
$$

where $\alpha=R / L$ (neper frequency) and $\omega_{0}=1 /(L C)^{1 / 2}$. We have also used the fact that $d V_{D} / d t=1$. The ratio of neper frequency and resonance frequency gives the damping factor $\xi$, which is

$$
\xi=\frac{\alpha}{\omega_{0}}
$$


Depending on the value of the damping factor, there are three different solutions for Eq. (4), representing different damped situations respectively. When the damping factor is lower than 1 , the solution, shown as Eq. (6), has a sinusoidal function, which enables the current oscillation in the RLC module.

$$
I_{R L C}=A_{R L C} e^{-\alpha t} \cos \left(\omega_{d} t+\varphi\right)+L C,
$$

where $A_{R L C}$ and $\varphi$ are coefficients determined by initial conditions, $t$ is the time parameter, and the frequency $\omega_{d}$ is given by

$$
\omega_{d}=\sqrt{\omega_{0}^{2}-\alpha^{2}} \text {. }
$$

From Eq. (6), we can see that the oscillating current is proportional to the exponential coefficient $e^{-\alpha t}$. Usually, with a positive $\alpha$, this exponential coefficient leads to decay in the oscillating current. However, a growth can be expected with a negative $\alpha$, on the contrary. As reported in literature, negative resistance is observed quite often in conducting polymers [12], resistance switching materials [13] and unijunction transistors [14]. Since we are investigating the electrochemical transistor with the conducting polymer PEDOT:PSS which's resistance varies with its redox states, it is reasonable to introduce an equivalent negative resistor in this model. Therefore, we assign the resistor $R$ in RLC module to act as the negative resistor, to keep this model simple and straightforward. Then, self-starting oscillations can be expressed by Eq. (8) with this assumption.

To address the channel current $I_{R T}$, gate voltage dependent channel resistance is presented below. Generally, electrochemical transistors are normally-on [3, 15], which is similar to junction gate field effect transistors working in depletion mode. Applying bias voltage to the gate, the channel is switched off, so-called "pinched". This is due to the reduction process of PEDOT:PSS by ascending gate voltage in positive direction. There are only a few examples to model the static behavior of electrochemical transistors. For instance, a model has been reported based on the 
spatially doping/de-doping process in electrochemical transistors [15]. However, we intend to avoid much complexity in this part and keep the model as compact as possible. Hence, here we assume the channel resistance $R_{T}$ is approximately dependent on the quantity of PEDOT obtained by reducing PEDOT $^{+}$ions, thus

$$
R_{T}=\frac{R_{0}}{1-\kappa+\lambda}, \quad 0<\kappa<1
$$

where $\kappa=V_{G} / V_{P}$ is the percentage of $\mathrm{PEDOT}^{+}$ions reduced, $V_{P}$ is the gate voltage that makes the $\mathrm{PEDOT}^{+}$fully reduced and $\lambda$ controls the resistance of $\mathrm{PEDOT}^{+}$when fully reduced.

From Fig. 2(b), the differential current is approximately proportional to the time/drain voltage, as mentioned above. Taking this into account, $I_{R T}$ can be expressed as

$$
I_{R T}=\frac{V_{D}^{2}}{R_{0} /\left(1-V_{G} / V_{P}+\lambda\right)} .
$$

Summing of $I_{R L C}$ and $I_{R T}$, the drain current is given by following formula:

$$
I_{D}=\frac{V_{D}^{2}}{R_{0} /\left(1-V_{G} / V_{P}+\lambda\right)}+A_{R L C} e^{-\alpha t} \cos \left(\omega_{d} t+\varphi\right)+L C
$$

\section{Results and discussion}

Fig. 4 presents the simulated results, exhibiting highly similar features to the experimental data. The model parameters are $R=-15 \Omega, L=3 \mathrm{H}, C=3 \times 10^{-5} \mathrm{~F}, R_{0}=1 \times 10^{4} \Omega$, and $\lambda=0.15$, respectively. The coefficients $A_{R L C}=\operatorname{sqrt}\left(i_{0}{ }^{2}+\left(i_{0}{ }^{\prime}+\alpha i_{0}\right)^{2} /\left(\omega_{d} i_{0}\right)^{2}\right)$ and $\varphi=-\arctan \left(\left(i_{0}{ }^{\prime}+i_{0}\right) /\left(\omega_{d} i_{0}\right)\right)$ are determined by the initial current $i_{0}$ and the initial current varying rate $i_{0}$ '. Here, the initial current is considered to be null for the first $V_{D}$ sweeping and the current at the end is assumed to be the following initial current for the next $V_{D}$ sweeping, and the initial current varying rate is assumed to be constant $\left(1 \times 10^{-9} \mathrm{~A} / \mathrm{s}\right)$. From the above equations, it can be concluded that the $A_{R L C}$ and $\varphi$ are very crucial to the amplitude and phase of oscillation. The assumption of varied initial current brings a very slight phase shift and a rising amplitude to simulation curves. As it is clear from the figure, this 
model explains the self-starting oscillation in PEDOT:PSS electrochemical transistors. However, it does not show a complete match between the experimental data and simulation results, because the real device behavior could be more complicated than that of the equivalent circuit presented here. Another feature worthy to note is the oscillation frequency, which is up to around $10 \mathrm{~Hz}$ in PEDOT:PSS electrochemical transistors. Comparing with those in other polymer electronic devices (less than $1 \mathrm{~Hz}$ [6], or even as low as $\sim 0.005 \mathrm{~Hz}$ [5]), this is a quite high value, although it cannot compete with conventional inorganic oscillators. This suggests that PEDOT:PSS electrochemical devices are promising for high performance polymer oscillators along with some particular properties, such as printability, flexibility, and even color switching. Furthermore, the presence of "inductor-like" element enables the possibility to implement other analog functions (filter, voltage multiplier, etc.) by PEDOT:PSS electrochemical devices.

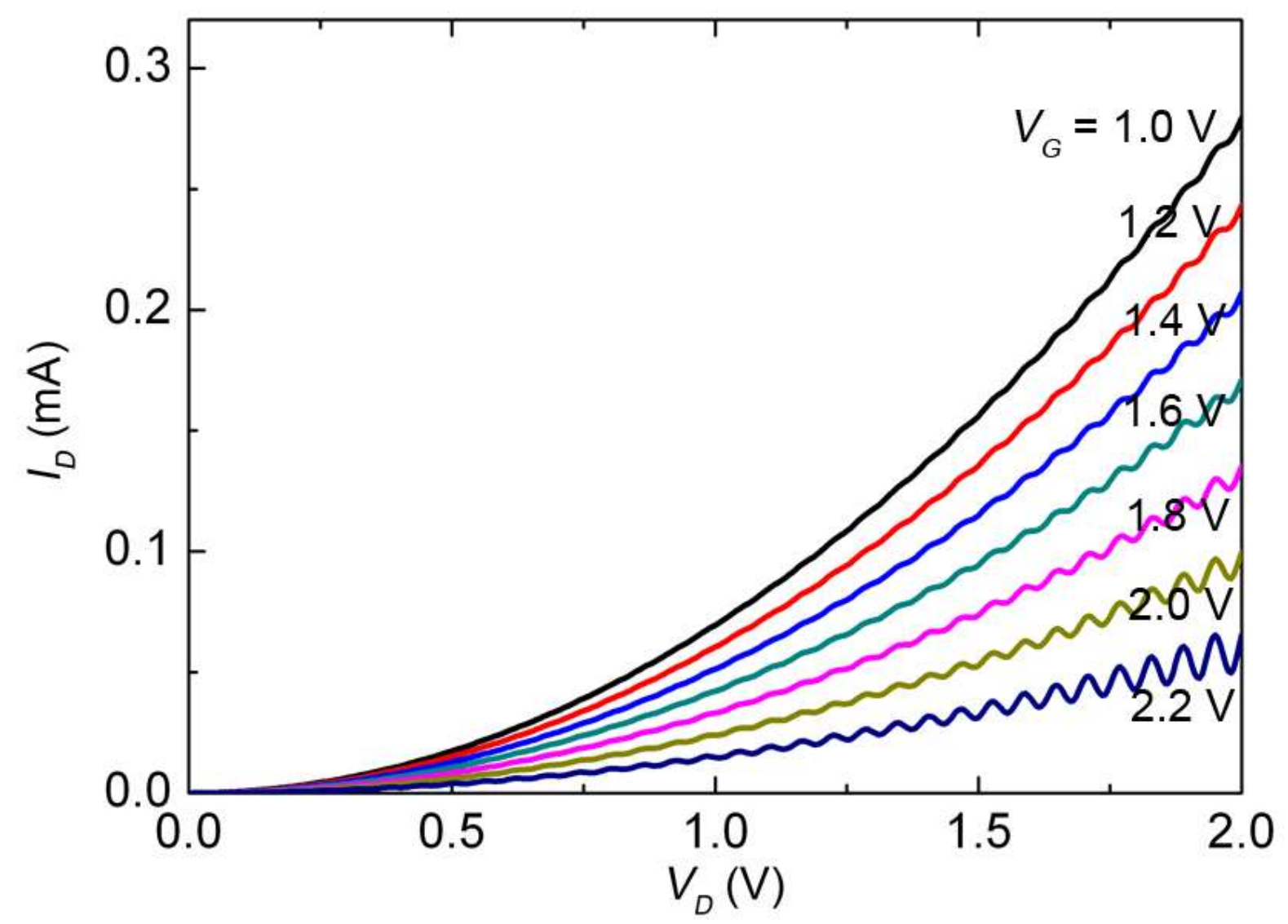

Fig. 4 The output curves given by the simulation results, which show good agreement in appearance with the experimental data. 
Besides oscillators, the PEDOT:PSS electrochemical transistors also can work as normal transistors in depletion mode. When the damping factor of RLC module is higher than one, this overdamped RLC circuit cannot generate any oscillation at all. Even if the RLC circuit works in the underdamped state, the amplitude is still controllable, as referred above. As long as the gate voltage is low enough, the amplitude can be suppressed to be neglectable. This is consistent with what we have observed in experiments that the current oscillation only occurred in some devices with particular geometry, which may correspond to the exact parameters in the model to generate oscillating current. The unclear connection between different device behaviors and the device geometry is quite complex and is still under investigation.

This compact model provides a good correspondence qualitatively to the experimental data, although there are only a few parameters in this model. However, the electrochemical transistors have too many uncertainties to be identified, associated with electron/ion transport and chemical reaction. This is why more measurements on PEDOT:PSS electrochemical transistors are currently conducted to refine the model. Another ongoing subject is to develop an efficient and straightforward methodology to extract parameters, which is very important to simulate analog functions at circuit level. The Belousov-Zhabotinsky reaction [16], which is a purely chemical reaction, is cyclic so that it can be regarded as a chromatic oscillation at slow rate. It is likely that there is something in common with the current oscillations in electrochemical transistors, although it is still unidentified. It is believed that one of very important features of living systems is the rhythmic process, which exhibits similar behaviors with chemical oscillation. This implies that electrochemical devices acting as oscillators could play an important role for artificial biological components, such as neurons [17].

\section{Conclusions}

In summary, a compact model based on an RLC circuit is developed to describe the electrical characteristics of the PEDOT:PSS electrochemical transistors. In particular, an 
unexpected current oscillation in the transistors can be well explained, by introducing an inductor module in the equivalent circuit. The simulation results show good agreement with the experimental data. By parameter modulation, the oscillation amplitude diminishes and the electrochemical device can be operated as a normal transistor. The oscillation can also be eliminated by adjusting device parameters to work in overdamped/critical damped mode. The model predicts that PEDOT:PSS electrochemical transistors are promising for analog circuit functions and even artificial biological systems.

\section{Acknowledgments}

The authors gratefully thank Dr. David Nilsson and Dr. Peter Andersson Ersman from Acreo for fruitful discussions and the Strategic Research Foundation SSF for financial support under the “OPEN” project. 


\section{References}

1 H. Sirringhaus, T. Kawase, R. H. Friend, T. Shimoda, M. Inbasekaran, W. Wu, E. P. Woo, Science 290 (2000) 2123-2126.

2 M. Berggren, D. Nilsson and N. D. Robinson, Nature Mater. 6 (2007) 3-5.

3 D. Nilsson, N. Robinson, M. Berggren and R. Forchheimer, Adv. Mater. 17 (2005) 353-358.

4 R. Mannerbro, M. Ranlöf, N. Robinson and R. Forchheimer, Synth. Metals 158 (2008) 556560.

$5 \quad$ A. Smerieri, V. Erokhin and M. P. Fontana, J. Appl. Phys. 103 (2008) 094517.

6 J. A. Freire, G. A. Dal Moro, R. Toniolo, I. A. Hümmelgen and C. A. Ferreira, Org. Electron. 7 (2006) 397-402.

7 F. A. Castro, P. R. Bueno, C. F. O. Graeff, F. Nüesch, L. Zuppiroli, L. F. Santos and R. M. Faria, Appl. Phys. Lett. 87 (2005) 013505.

8 E. Ehrenfreund, C. Lungenschmied, G. Dennler, H. Neugebauer and N. S. Sariciftci, Appl. Phys. Lett. 91 (2007) 012112.

9 L. S. C. Pingree, B. J. Scott, M. T. Russell, T. J. Marks and M. C. Hersam, Appl. Phys. Lett. $86(2005) 073509$.

10 G. Garcia-Belmonte, J. Bisquert, P. R. Bueno, C. F. O. Graeff, and F. Castro, Synth. Metals 159 (2009) 480-486.

11 M. Ershov, H. C. Liu, L. Li, M. Buchanan, Z. R. Wasilewski and A. K. Jonscher, IEEE Trans. Electron Devices 45 (1998) 2196-2206.

12 D. Jeon, J. Kim, M. C. Gallagher and R. F. Willis, Science 256 (1992) 1662-1664.

13 M. D. Pickett, J. Borghetti, J. J. Yang, G. Medeiros-Ribeiro and R. S. Williams, Adv. Mater. 23 (2011) 1730-1733.

14 S. Bellone, S. Daliento and A. Sanseverino, Solid-State Electronics 45 (2001) 483-488. 
15 D. A. Bernards and G. G. Malliaras, Adv. Funct. Mater. 17 (2007) 3538-3544.

16 L. Treindl, T. Hemmingsen and P. Ruoff, Chem. Phys. Lett. 269 (1997) 263-267.

17 T. Berzina, A. Smerieri, M. Bernabò, A. Pucci, G. Ruggeri, V. Erokhin and M. P. Fontana, J. Appl. Phys. 105 (2009) 124515. 